\title{
The VSOP Project: Space VLBI Imaging of AGN at 1.6 and $5 \mathrm{GHz}$
}

\author{
Y. Murata, H. Hirabayashi, P.G. Edwards \\ Institute of Space and Astronautical Science, 3-1-1 Yoshinodai, \\ Sagamihara, Kanagawa 229-8510, Japan
}

\begin{abstract}
The VLBI Space Observatory Programme (VSOP) combines an orbiting radiotelescope with arrays of ground radio telescopes to extend the Very Long Baseline Interferometry (VLBI) technique to baselines up to almost three Earth diameters. In this paper, we present results from VSOP observations of active galactic nuclei (AGN) at 1.6 and $5 \mathrm{GHz}$ from the first 3.5 years of the mission.
\end{abstract}

\section{The VSOP misson}

The VSOP mission started from the launch of the HALCA (Highly Advanced Laboratory for Communications and Astronomy) satellite on February 12, 1997. Observations started in May 1997, and were soon followed by the detection of the first fringes between HALCA and ground radio-telescopes. Although HALCA has experienced some troubles (such as the low gain of $22 \mathrm{GHz}$ receiver and failure of one reaction wheel), VSOP observations have continued for well over three years. It is expected that the solar battery power, which was thought would be the deciding factor in HALCA's lifetime, is sufficient to continue observing for 2-3 more years (Murata et al. 2000). If there are no unexpected on-board failures, Space VLBI observations will be able to continue for at least the nominal 5 year mission lifetime.

The VLBI Space Observatory Programme is a very international mission (Hirabayashi et al. 1998, 2000a). The HALCA satellite was built and launched in Japan, and is supported by five tracking stations and one commanding station in four countries, three correlators in three countries, and 32 telescopes in 14 countries! Thanks to the efforts of those operating these many critical elements, a wealth of scientific results have been possible from VSOP observations. Proceedings from the COSPAR meeting in July 1998, and VSOP Symposium in January 2000 illustrate the variety of science being undertaken with VSOP observations. We focus here on VSOP observations of active galactic nuclei.

\section{VSOP and HALCA properties}

HALCA is effectively an $8 \mathrm{~m}$ diameter antenna in an orbit with a $21400 \mathrm{~km}$ apogee height and $560 \mathrm{~km}$ perigee height. It has three observing bands: from 1.60-1.73 GHz (L-band), 4.7-5.0 GHz (C-band) and 22.0-22.3 GHz (K-band). The $22 \mathrm{GHz}$ band was found after launch to have an unexpectedly low gain 
and so is not used for routine scientific observing. The 1.6 and $5.0 \mathrm{GHz}$ bands have typical system noise temperatures of $75 \pm 30 \mathrm{~K}$ and $95 \pm 30 \mathrm{~K}$, respectively (Kobayashi et al. 2000).

Baseline sensitivities of $80 \mathrm{mJy}$ at L-band and $100 \mathrm{mJy}$ at $\mathrm{C}$ band are achievable, for a $7 \sigma$ detection with a VLBA $25 \mathrm{~m}$ antenna. Typical resolutions for VSOP observations at 1.6 and $5.0 \mathrm{GHz}$ are 0.75 mas and 0.25 mas, respectively. The $5.0 \mathrm{GHz}$ band resolution is almost equivalent with that for $15 \mathrm{GHz}$ observations with the VLBA. Thus we can study the appearance of astronomical objects with similar resolutions at two different frequencies and can determine the spectral index variation over the source. Such observations are a powerful tool to understanding the physical properties of the source observed.

\section{Scientific Results}

\subsection{Polarization Observation}

Although HALCA has only one polarization receiver system (LCP only), with dual polarisation ground observations VSOP can make polarisation observations to make high resolution studies of spatial variations in magnetic field structures. VSOP polarization observations (Gabuzda, 1999) revealed a twisted jet in the AGN 1803+784, with the magnetic field remaining perpendicular to the jet direction all along the bent structure. A detailed study of VSOP polarization observations is made in Kemball et al. (2000).

\subsection{Monitoring Observation}

Multi-epoch observations have been made of a number of key sources. For most sources, however, because of HALCA's sun-angle constraints, there are periods when observations are not possible. However, sources near the ecliptic poles are visible all year round and so can be monitored at regular intervals. An example is $1928+783$, for which changes in the milli-arcsecond-scale structure have been studied by Murphy et al. (2000).

\subsection{High Linear Resolution Studies}

For low redshift sources, VSOP's high angular resolution translates into high linear resolution. For M87, the VSOP beam at $5 \mathrm{GHz}$ is $\sim 300$ times the Schwarzschild radius of the super-massive black hole revealed by HST spectral studies. VSOP observations of M87 are enabling quantitative studies of how jet sub-structure evolves with time. The first VSOP image at $1.6 \mathrm{GHz}$ showed a helical structure (Reid et al. 2000). A more recent, higher sensitivity, $1.6 \mathrm{GHz}$ observation made in March 2000 (Reid et al. private communication) shows limb-brightened structure of the jet. VSOP monitoring observations at $5 \mathrm{GHz}$ do not show the $6 c$ movement which is seen in the outer jets by the HST (Junor et al. 2000).

\subsection{Spectral Index Mapping and Free-Free Absorption}

VSOP has the same resolution as ground VLBI with three times higher frequency. Spectral mapping with matched resolution allows information on the optical thickness, spectral aging, etc. to be determined. The sources studied 
with this technique include 3C 279 (Piner et al. 2000), 3C 84 (Asada et al. 2000) and Mkn 501 (Edwards et al. 2000).

Free-free absorption of radiation from the counter-jet of NGC 4261 in the sub-parsec accretion disk was clearly seen with the use of a $4.9 \mathrm{GHz}$ VSOP image and ground-based VLBI images (Jones et al. 2000). Similar studies have been made of a number of GPS sources also (Snellen et al. 2000; Kameno et al. 2000a, 2000b, Sutou et al. 2000).

\subsection{X-ray Jets}

The synchrotron photons at radio wavelengths are intimately related to $\mathrm{X}$ - and gamma-rays by the inverse-Compton effect. A second-epoch VSOP observation of PKS 0637-752 was made in August 1999, coordinated with a calibration observation of the just-launched Chandra satellite. The Chandra observation revealed an X-ray jet consistent with the ATCA radio images (Schwartz et al. 2000). The VSOP observations were used to establish an $\sim 11$ c super-luminal motion in the parsec-scale jet, placing strong constraints on the angle between the parsec-scale jet and our line of sight to the quasar (Tingay et al. 2000, Lovell et al. 2000).

\subsection{Quasars at High Redshift}

Imaging of high-redshift quasars at the VSOP frequencies of 1.6 and $5 \mathrm{GHz}$ enables their milli-arcsecond radio structures to be studied at emitted frequencies of 6.5-8.0 and $20-27 \mathrm{GHz}$ respectively. Over 20 VSOP observations of high$z$ quasars have been conducted to date. Highlights include the detection of extremely violent bending in the quasar 1351-017 $(z=3.71)$ and the discovery of the most distant rich "core-jet" structure in the quasar $2215+020$ at $z=3.55$ (Gurvits et al. 2000).

\subsection{High Brightness Temperature Sources}

There is a brightness temperature limit of $\sim 10^{12} \mathrm{~K}$ for incoherent synchrotron emission in the rest frame of the emitting plasma due to inverse Compton scattering loss of the emitting particles (Kellermann and Pauliny-Toth 1969). The brightness temperature observed in our reference frame differs from its value in the emitted frame by the factor $\delta /(1+z)$, where $\delta$ is the Doppler factor of the component in the rest frame of the radio source core. VSOP is a unique instrument for the measurement of brightness temperature, as the brightness temperature is directly proportional to the maximum baseline length sampled in the observation. Brightness temperatures in excess of $10^{12} \mathrm{~K}$ have been measured for a number of sources implying large Doppler boosting factors for material in the jets (e.g., Bower \& Backer 1998, Shen et al. 1999, Preston et al. 2000).

\subsection{The VSOP Survey Program}

The VSOP Survey Program is providing a large complete sample of homogeneous data on the sub-milli-arcsecond radio structures of 289 AGN, which is essential for studying cosmology and statistics of AGN, planning future VLBI observations, and for designing future Space VLBI missions (Hirabayashi et al. 2000b). 


\section{Summary and the Future}

VSOP observations have produced the highest resolution 5 and $1.6 \mathrm{GHz}$ images, advancing our understanding of AGN on a number of fronts. Space VLBI observations at $22 \mathrm{GHz}$ and higher frequencies with the future missions hold a great deal of promise.

Acknowledgments. We gratefully acknowledge the many organizations and astronomers around the world who have participated in VSOP observations.

\section{References}

N.B. APRSV2000 denotes Astrophysical Phenomena Revealed by Space VLBI, ed. H. Hirabayashi, P.G. Edwards \& D.W. Murphy (Sagamihara: ISAS)

Asada, K., Kameno, S., Inoue, M. et al. 2000, in APRSV2000 p. 51

Bower, G.C. \& Backer, D.C. 1998, ApJ, 507, L117

Edwards, P.G., Giovannini, G., Cotton, W.D. et al. 2000, PASJ, 52, in press

Gabuzda, D. 1999, New Astronomy Reviews, 43, 691

Gurvits, L.I., Frey, S., Schilizzi, R.T. et al. 2000, Adv. Space Res., 26, 719

Hirabayashi, H., Hirosawa, H., Kobayashi, H., et al. 1998, Science, 281, 1825 and erratum 282, 1995

Hirabayashi, H., Hirosawa, H., Kobayashi, H. et al. 2000a, PASJ, 52, in press

Hirabayashi, H., Fomalont, E.B., Horiuchi, S., et al. 2000b, PASJ, 52, in press

Jones, D.L., Wehrle, A.E., Piner, B.G. et al. 2000, in APRSV2000, p. 71

Junor, W., Biretta, J.A., Owen, F.N. \& Begelman, M.C. 2000, in APRSV2000 p. 13

Kameno, S., Sawada-Satoh, S., Shibata, K. et al. 2000a, in APRSV2000, p. 87

Kameno S., Horiuchi S., Shen Z.-Q., Inoue M., et al. 2000b, PASJ, 52, 209

Kellermann, K.I. \& Pauliny-Toth, I.I.K. 1969, ApJ, 155, L71

Kemball, A., Flatters, C., Gabuzda, D., et al. 2000, PASJ, 52, in press

Kobayashi, H., Wajima, K., Hirabayashi, H. et al. 2000 PASJ, 52, in press

Lovell, J.E.J., Tingay, S.J., Piner, B.G. et al. 2000, in APRSV2000, p. 215

Murata, Y., Hirabayashi, H., Kobayashi,H., et al. 2000, in Radio Telescopes, ed. H.R. Butcher, Proc. of SPIE Vol 4015, p. 204

Murphy, D.W., Preston, R.A., Polatidis, A. et al. 2000, in APRSV2000, p. 47

Piner, B.G., Edwards, P.G., Wehrle, A.E. et al. 2000, ApJ, 537, 91

Preston, R.A., Lister, M.L., Tingay, S.J. et al. 2000, in APRSV2000, p. 199

Reid, M. et al. 2000, in preparation

Schwartz, D.A. et al. 2000, ApJ, 540, L69

Shen, Z.-Q., Edwards, P.G., Lovell, J.E.J. et al. 1999, PASJ, 51, 513

Snellen, I., Tschager, W., Schilizzi, R. et al. 2000, in APRSV2000, p. 79

Sudou,H., Taniguchi,Y., Ohyama,Y. et al. 2000, PASJ, 52, in press

Tingay, S.J., Jauncey, D.L., Reynolds, J.E. et al. 2000, Adv. Sp. Res., 26, 677 